\title{
Papillary fibroelastoma in a 63-year-old asymptomatic patient with paroxysmal atrial fibrillation
}

\author{
Corresponding author: \\ Witold Żurański, Department \\ of Cardiology and Internal Medicine, \\ Collegium Medicum in Bydgoszcz, \\ Nicolaus Copernicus University, Poland, \\ e-mail: witold.zuranski@gmail.com
}

Medical Research Journal 2020; Volume 5, Number 3, 215-218 10.5603/MRJ.a2020.0029 Copyright (C) 2020 Via Medica ISSN 2451-2591

\begin{abstract}
Papillary fibroelastoma (PFE) is the second most frequent benign cardiac tumor. It is located mainly in the heart valves. Because its course is usually clinically silent it is often detected accidentally in transthoracic echocardiography (TTE). Despite the benign nature of the lesion, the histological structure of PFE increases the risk of embolic episodes. Development of imaging techniques as well as their wider accessibility, especially TTE, contributes to the more common detection of this tumor in the general population. In this publication, we present a case of an asymptomatic 63-year-old woman in whom an aortic valve lesion resembling PFE was detected in TTE.
\end{abstract}

Key words: papillary fibroelastoma, cardiac tumor, paroxysmal atrial fibrillation, heart valves, embolic episodes

Med Res J 2020; 5 (3): 215-218

\section{Introduction}

Cardiac tumors are rare. They are classified as primary (benign or malignant), and secondary (metastases) that occur 20-40 times more frequently. The prevalence of primary cardiac tumors ranges from $0.001 \%-0.03 \%$ [1]. Among them $75 \%$ are benign, which include PFE- the most common cardiac valves tumor, that is diagnosed in as many as $75 \%$ of such cases [4]. It is most often localized within the aortic valve (29\%) [7]. Due to more frequent performing of transthoracic echocardiography (TTE) in the elderly population, the average age of detection of PFE is 60 years, but the tumor occurs in a wide age range (from newborns to elderly). It affects women and men equally often.

\section{Case report}

A 63-year-old female patient was admitted to the Department of Cardiology and Internal Medicine of University Hospital No. 1 in Bydgoszcz due to a suspected heart tumor. In outpatient TTE an additional echo in connection with the right coronary cusp of the aortic valve was visualized in the left ventricle outflow tract. The patient's past medical history included paroxysmal atrial fibrillation, hypertension, subclinical hyperthyroidism, right internal carotid aneurysm, multifocal cerebrovascular damage, and nicotinism. On admission, patient did not present stenocardial symptoms nor fever. Inflammation markers were within the normal range. Physical examination and other laboratory tests did not reveal abnormalities. The patient was on statin, aspirin, and thiamazole, without chronic anticoagulant therapy. Due to the CHAD2DS2-VASc score of 2 anticoagulation with apixaban $5 \mathrm{mg}$ twice daily was introduced to the treatment. During the hospitalization blood cultures were collected for infective endocarditis - all returned negative. Transoesophageal echocardiography (TEE) revealed additional, mobile echo $(1.2 \mathrm{~cm} \times 0.7 \mathrm{~cm})$ on a pedicle in connection with the right coronary cusp of the aortic valve (Fig. 1), without left ventricular outflow tract obstruction (LVOTO). Cardiac magnetic resonance (CMR) did not reveal a pathological mass in the aortic bulb nor the left ventricle outflow tract. Computed tomography (CT) of the heart with a submillimeter layer was then performed. It confirmed the presence of a mass sized $2.5 \mathrm{~cm} \times 4.0 \mathrm{~cm} \times 5.5 \mathrm{~cm}$ on the ventricular surface of the right aortic cusp, which penetrated the left ventricle outflow tract and balloted together with the cusp (Figure 2). However, the abovementioned diagnostic tests did not allow to determine the nature of the lesion. Despite the asymptomatic clinical course, the patient was qualified for urgent 

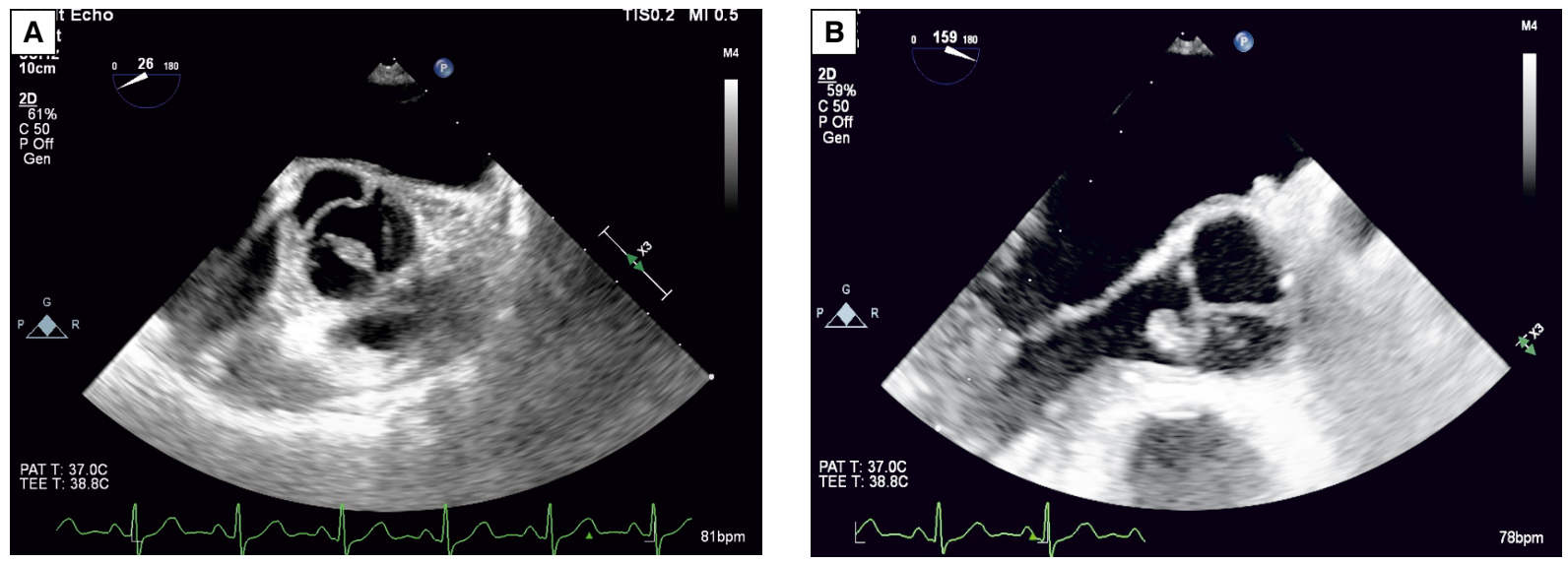

Figure 1. Papillary fibroelastoma in transesophageal echocardiography: A. midesophageal short axis of aortic valve view B. left ventricular outflow tract, long axis view. Echocardiographic parameters: LVEDD: $42 \mathrm{~mm}$, LA: $33 \mathrm{~mm}$, RV: $26 \mathrm{~mm}$, LVEF: 60\%, IVS: $11 \mathrm{~mm}$, PW: $11 \mathrm{~mm}$, aortic PG max: $14 \mathrm{~mm} \mathrm{Hg}$ LVEDD — left ventricular end-diastole diameter; LA — left atrium; RV — right ventricle; LVEF — left ventricular ejection fraction; IVS — interventricular septum thickness; PW — posterior wall thickness; PG max — maximum pressure gradient
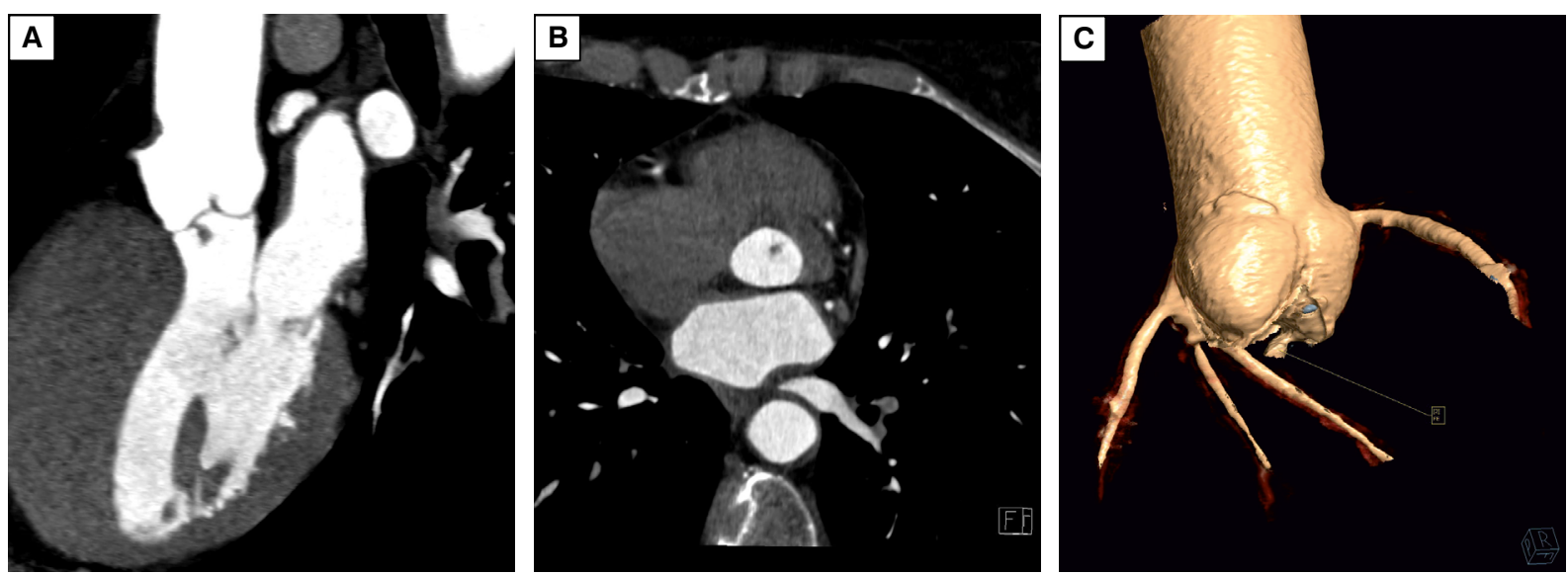

Figure 2. Papillary fibroelastoma of the heart. A. Cardiac computed tomography three-chamber view showing a polypoid lesion attached to the right coronary cusp. B. Axial reformatted image from conducted computed tomography demonstrates a small mass arising from the right coronary cusp. C. 3D-reconstruction showing papillary fibroelastoma attached to the right coronary cusp (yellow arrow). CT parameters: LVEF: 68 \%; LVEDV: $106 \mathrm{ml}$; LVESV: $34 \mathrm{ml}$; SV: $72 \mathrm{ml}$

CT — computed tomography; LVEF — left ventricular ejection fraction; LVEDV — left ventricular end-diastolic volume; LVESV — left ventricular end-systolic volume; SV — stroke volume

cardiac surgery. No significant stenoses were found in the routine pre-surgery coronary angiography. Next, the lesion was removed by aortotomy in the extracorporeal circulation. The tumor $(1.2 \mathrm{~cm} \times 0.7 \mathrm{~cm} \times 0.4 \mathrm{~cm})$ was excised completely without causing aortic valve defect, and after fixation in formalin, it was transferred to the Department of Clinical Pathomorphology of University Hospital No. 1 in Bydgoszcz for further examination. Light microscopy revealed a pedunculated lesion with numerous papillary branches made of acellular and avascular stroma covered with hyperplastic endocardial endothelium (Figure 3). Based on the microscopic assessment and clinical data, papillary fibroelastoma of the aortic valve has been diagnosed.

\section{Discussion}

PFE, after myxoma, is the second most common benign heart tumor [2, 3]. It is sometimes called as sea anemone, because of its undulating spines [5]. Its papillary protrusions are covered with regular endothelium and their interior is built of amorphous, dense tissue rich in collagen and elastic fibers. The layered structure 


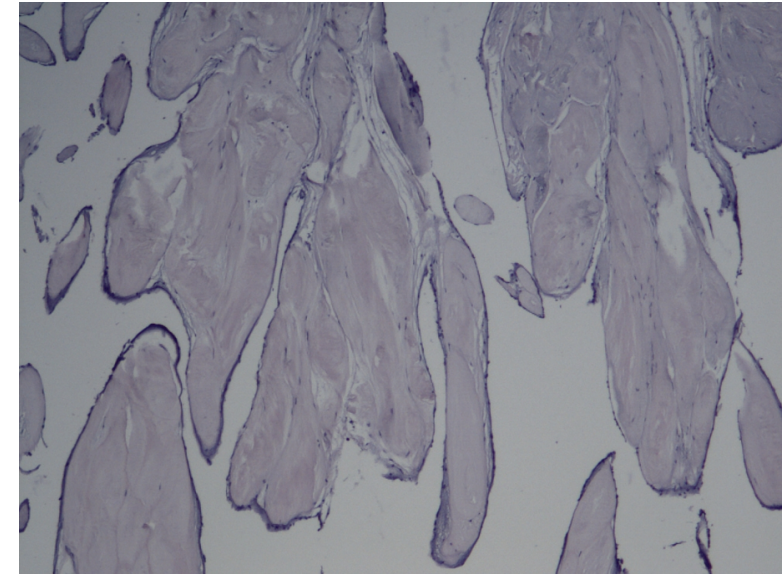

Figure 3. Histological structure of the reported papillary fibroelastoma

of the tumor resembles normal chordae tendineae [6]. PFEs are usually small tumors that develop in the heart chambers, occupying individual heart valves according to the following frequency: aortic valve (29\%), mitral valve (25\%), tricuspid valve (17\%) and pulmonary valve (13\%) [7]. Their diameter ranges from $2 \mathrm{~mm}$ to $70 \mathrm{~mm}$, with an average size of $9 \mathrm{~mm} \mathrm{[6].}$

The etiology of PFE is unclear and various possible causes have been proposed in the literature, such as prior damage to the endothelium, hamartomas and organized emboli. One of the most frequently quoted theories is the microthrombus theory, which states that tumors are acquired rather than congenital. [8, 9].

Although PFE histologically is a benign heart tumor, it can cause life-threatening embolic complications such as stroke, peripheral embolism, and myocardial infarction. Pulmonary embolism, congestive heart failure, ventricular fibrillation, syncope and sudden cardiac death are rare, but also have been reported [2]. Although the most common location of PFE are heart valves, it rarely causes their dysfunction [10].

PFE can be easily detected by echocardiography. TTE is sufficient as a screening test, however, TEE is the preferred method due to its higher resolution and better imaging capability [11]. Given that PFE is an avascular tumor, the imaging of choice, in this case, is CMR. However, the sensitivity of CMR in PFE imaging depends on the imaging technique used (the appropriate number of projections, sequential gating, ECG gating). Although there have been significant improvements in CMR and CT assessment of the cardiac valves, the time and spatial resolution are often inadequate to diagnose PFE due to its usually small size and mobile nature. This cause limitations of advanced imaging techniques in the diagnosis of the PFE.

On the basis of the described case, the authors noticed that there was a large discrepancy between the size of the tumor described in TEE and CT and the actu- al size of the tumor found after surgical removal. Several CT technologic advances e.g. submillimeter detector arrays increased rows of detectors, ECG gating- have resulted in improved imaging of cardiac structures, including cardiac masses. The current limitations of this technique include, in addition to the significant radiation dose, a lower temporal resolution compared to echocardiography and cardiac MRI. Moreover, CT is inferior to echocardiography in imaging small moving structures, because it does not allow real-time imaging and the imaging planes are limited to those allowed by the angulation of the gantry [14] .

Most PFEs are asymptomatic. Currently, there is no consensus on the treatment strategy of patients with PFE. Therapeutic decisions should take into account the severity of symptoms, location and size of the mass. Generally, symptomatic lesions should be treated by surgical excision of the tumor. Asymptomatic patients with lesions greater than $1 \mathrm{~cm}$, that are mobile, and especially left-sided are also eligible for surgical resection because of the high risk of thromboembolic complications. However, if the masses are small, non-mobile, located on the left side of the heart, frequent echocardiographic assessment is sufficient. Small right-sided changes also should be monitored only. Despite the fact that no data exist on the evaluation of the efficacy of anticoagulation or antiplatelet therapy in patients with PFE, tumor structure requires consideration of this treatment in patients treated conservatively $[6,12,13]$.

This case report shows that more advanced imaging examinations (CMR and MDCT) are not always sensitive enough and TTE and TEE may constitute the basis for qualification for cardiac surgery.

\section{Conclusions}

TTE is a useful tool for screening and the initial diagnosis of a cardiac tumor. For further diagnosis or in the case of conflicting test results, other imaging methods should be performed (e.g. TEE, CMR, MDCT). The results obtained from imaging examinations have a direct impact on the choice of treatment. In particular, PFE needs to be differentiating from thrombi and cardiac myxomas because of the differences in their treatment. A clear surgical margin is necessary for the excision of a myxoma due to its high rate of recurrence. PFE rarely recur after resection, and so it is recommended to preserve the valvular function by shaving off the tumor. Although the TTE is widely used to screen for fibroelastomas, the TEE is more sensitive and can provide a higher resolution imaging for surgical planning. The importance of CMR and MDCT as tools in differential diagnosis of soft tissue masses of the heart is currently under investigation. 


\section{Acknowledgements}

The authors would like to acknowledge Piotr Adamski MD, Ph.D. (Department of Cardiology and Internal Medicine, Collegium Medicum, Nicolaus Copernicus University, Bydgoszcz, Poland) for his contributions in editing this manuscript, Przemysław Ratajczak MD (Department of Radiology and Diagnostic Imaging, Nicolaus Copernicus University, Collegium Medicum, Bydgoszcz, Poland) and Marek Zdrenka MD (Department of Clinical Pathomorphology, Collegium Medicum, Nicolaus Copernicus University, Bydgoszcz, Poland) and) for sharing images.

\section{Conflicts of Interest}

The authors declare that there is no conflict of interest regarding the publication of this paper.

\section{References:}

1. Butany J, Nair V, Naseemuddin A, et al. Cardiac tumours: diagnosis and management. The Lancet Oncology. 2005; 6(4): 219-228, doi: 10.1016/s1470-2045(05)70093-0.

2. R R, et al. Gopaldas, P. V. Atluri, A. S. Blaustein, , "Papillary fibroelastoma of the aortic valve: operative approaches upon incidental discovery, " Texas Heart Institute Journal, vol. 36, no 2, pp 160-163. ; 2009.
3. Bossert T, Gummert JF, Battellini R, et al. Surgical experience with 77 primary cardiac tumors. Interact Cardiovasc Thorac Surg. 2005; 4(4): 311-315, doi: 10.1510/icvts.2004.103044, indexed in Pubmed: 17670419.

4. Paraskevaidis IA, Michalakeas CA, Papadopoulos $\mathrm{CH}$, et al. Cardiac tumors. ISRN Oncol. 2011; 2011: 208929, doi: 10.5402/2011/208929, indexed in Pubmed: 22091416.

5. Aryal MR, Badal M, Mainali NR, et al. Papillary fibroelastoma of the aortic valve: An unusual cause of angina. World J Cardiol. 2013; 5(4): 102-105, doi: 10.4330/wjc.v5.i4.102, indexed in Pubmed: 23675556.

6. Chitwood WR. Cardiac neoplasms: current diagnosis, pathology, and therapy. J Card Surg. 1988; 3(2): 119-154, doi: 10.1111/j.15408191.1988.tb00232.x, indexed in Pubmed: 2980011.

7. Grinda JM, Couetil JP, Chauvaud S, et al. Cardiac valve papillary fibroelastoma: surgical excision for revealed or potential embolization. J Thorac Cardiovasc Surg. 1999; 117(1): 106-110, doi: 10.1016/s00225223(99)70474-5, indexed in Pubmed: 9869763.

8. MAGAREY FR. On the mode of formation of Lambl's excrescences and their relation to chronic thickening of the mitral valve. J Pathol Bacteriol. 1949; 61(2): 203-8, 5 pl, doi: 10.1002/path.1700610207. indexed in Pubmed: 15392478.

9. Malik MF, Sagar K, Wynsen JC, et al. Evolution of a papillary fibroelastoma. J Am Soc Echocardiogr. 1998; 11(1): 92-94, doi: 10.1016/s08947317(98)70130-0, indexed in Pubmed: 9487480.

10. Parthenakis F, Nyktari E, Patrianakos A, et al. Asymptomatic papillary fibroelastoma of the aortic valve in a young woman - a case report. Cardiovasc Ultrasound. 2009; 7: 43, doi: 10.1186/1476-7120-7-43, indexed in Pubmed: 19725951.

11. Sun JP, Asher CR, Yang XS, et al. Clinical and echocardiographic characteristics of papillary fibroelastomas: a retrospective and prospective study in 162 patients. Circulation. 2001; 103(22): 2687-2693, doi: 10.1161/01.cir.103.22.2687, indexed in Pubmed: 11390338

12. Bruce CJ. "Cardiac tumors: diagnosis and management, " Heart, vol. 97, no 2, pp 151-160. ; 2011

13. Baikoussis NG, Dedeilias $P$, Argiriou M, et al. Cardiac papillary fibroelastoma; when, how, why? Ann Card Anaesth. 2016; 19(1): 162-165, doi: 10.4103/0971-9784.173040, indexed in Pubmed: 26750694.

14. Gowda RM, Khan IA, Nair CK, et al. Cardiac papillary fibroelastoma: a comprehensive analysis of 725 cases. Am Heart J. 2003; 146(3): 404-410, doi: 10.1016/S0002-8703(03)00249-7, indexed in Pubmed: 12947356. 